\title{
Adherencia a la suplementación con micronutrientes y factores asociados en niños de 6 a 35 meses en Tacna
}

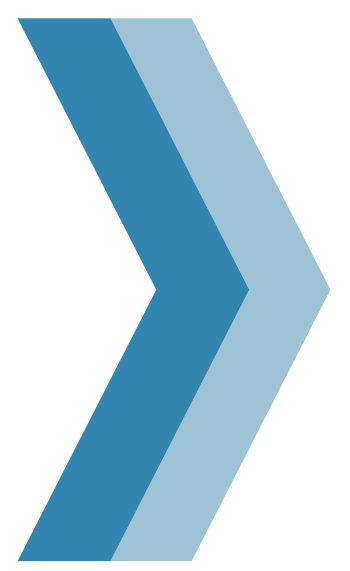

\author{
Adherence to supplementation with micronutrients and \\ associated factors in children aged 6 to 35 months in Tacna
}

\author{
Luzmeidy Fiorella Chambilla-Quispe ${ }^{\text {la }}$
}

${ }^{1}$ Centro de Salud Mental Comunitario Villa Tacna. Tacna, Perú.

${ }^{a}$ Enfermera asistencial, Licenciada en Enfermería con especialidad en Cuidado Enfermero en Crecimiento y Desarrollo.

\section{RESUMEN}

La Anemia es considerada un problema de salud pública grave, ya que tiene una prevalencia del 42,6 $\%$ en niños menores de 5 años según la Organización Mundial de la Salud (1); y en nuestro país, afecta al 43,5\% de niños según la Encuesta Demográfica y de Salud Familiar (ENDES) en el 2015 (2). Objetivo: Determinar la asociación entre la adherencia a la suplementación con micronutrientes y los factores asociados de la madre/cuidador del micronutriente y de la prestación de servicios de salud. Material y métodos: El estudio fue de tipo descriptivo, retrospectivo y de corte transversal. La población estuvo conformada por niños de 6 a 35 meses que estaban recibiendo micronutrientes. Resultados: El $67,1 \%$ de niños presentaron adherencia inadecuada a micronutrientes. Los factores identificados fueron: el $72,2 \%$ de madres tenían nivel educativo secundario, el $67,1 \%$ eran amas de casa y el 78,5\% se olvidaban de dar los micronutrientes; respecto al micronutriente, el $57 \%$ de niños presentaron efectos colaterales y al $43 \%$ no les agradaba consumirlo; respecto a la prestación de servicios de salud, el 97,5 $\%$ de madres manifestaron haber recibido explicación de cómo dar el micronutriente, el $81 \%$ recibió buen trato, el $62 \%$ fue atendida de inmediato y el 91,1\% afirmó buena disponibilidad del producto. Tras el análisis estadístico, se determinó asociación significativa entre la adherencia y el olvido de dar los micronutrientes con un $\mathrm{OR}=2,978$ y $\mathrm{p}$ valor $=0,000$; sin embargo, no se evidenció asociación significativa entre la adherencia y demás factores estudiados. Conclusiones: La adherencia se asocia significativamente con el olvido de dar los micronutrientes.

Palabras claves: Adherencia, factores asociados, suplementación con micronutrientes.

\section{ABSTRACT}

Anemia is considered a serious public health problem, since it has a prevalence of $42.6 \%$ in children under 5 according to the World Health Organization (1); and in our country, it affects to the $43.5 \%$ of children according to the Demographic and Family Health Survey (ENDES) in 2015 (2). Objective: To determine the association between adherence to micronutrient supplementation and the factors associated to the mother/caregiver of the micronutrient and the provision of health services. Material and methods: The study was descriptive, retrospective and cross-sectional. The population was made up of children aged 6 to 35 months who were receiving micronutrients. Results: $67.1 \%$ of children had inadequate adherence to micronutrients. The factors identified were: $72.2 \%$ of the mothers had secondary education, $67.1 \%$ were housewives and the $78.5 \%$ forgot to give micronutrients; regarding the micronutrient, $57 \%$ of the children presented side effects and $43 \%$ did not like to consume it; Regarding the provision of health services, $97.5 \%$ of the mothers said they had received an explanation of how to give the micronutrient, $81 \%$ received good treatment, $62 \%$ were treated immediately and $91.1 \%$ reported good product availability. After the statistical analysis, it was determined a significant association between adherence and forgetting to give micronutrients with an $\mathrm{OR}=2,978$ and $\mathrm{p}$ value $=0.000$; however, there was no significant association between adherence and other factors studied. Conclusions: Adherence is significantly associated with forgetting to give micronutrients.

Keywords: Adherence, associated factors, micronutrient supplementation. 


\section{Introducción}

La anemia es considerada un problema de salud pública grave por la Organización Mundial de la Salud, ya que en el 2015 se reportó que el 42,6 \% de niños menores de 5 años en el mundo tenían anemia (1). En nuestro país, según la Encuesta Demográfica y de Salud Familiar (ENDES), la prevalencia de anemia fue de $43,5 \%$ en el 2015 , siendo la deficiencia de hierro la causa principal de la mitad de casos de anemia (2). La anemia afecta el desarrollo cerebral del niño, lo cual puede ser irreversible si se presenta en los dos primeros años de vida, ya que este es el periodo crítico del desarrollo cerebral debido a que en este periodo se da el pico máximo de crecimiento y diferenciación cerebral (2).

El Ministerio de Salud del Perú aprobó una política de suplementación preventiva con micronutrientes para reducir la anemia (3). Sin embargo, no se la ha logrado disminuir como se desearía, lo que hace pensar en factores que limitan la efectividad de la suplementación y en otros que la favorecen, influyendo en su adherencia. Por esta situación, se ha visto la necesidad de determinar la asociación entre la adherencia a la suplementación con micronutrientes y los factores de la madre/cuidador del micronutriente y de la prestación de servicios de salud en niños de 6-35 meses.

\section{Material y métodos}

Estudio de tipo descriptivo, retrospectivo, de corte transversal. La población de estudio estuvo conformada por 425 niños de 6 a 35 meses de edad con sus respectivas madres/cuidadoras, las cuales se encontraban recibiendo micronutrientes en el Puesto de Salud "Cono Norte" del distrito de Ciudad Nueva. La muestra de trabajó fue de 79 niños elegidos por muestreo sistemático.

Para la recolección de datos de la variable dependiente Adherencia, se utilizó como técnica el análisis documental del libro de seguimiento de micronutrientes y la entrevista estructurada a la madre/cuidador del niño. Para medir la adherencia, se utilizó la fórmula que aplica el Ministerio de Salud (3). Siendo la adherencia adecuada cuando el consumo de micronutrientes era $>=90 \%$; $y$ adherencia inadecuada cuando el consumo era $<90 \%$. Respecto a la variable independiente Factores asociados se utilizó como técnica la encuesta y como instrumento el cuestionario. El cuestionario fue validado por expertos y tuvo un coeficiente de Alfa-Crombach de 0,751 indicando que sí era confiable.

\section{Resultados}

En la Tabla 1 se aprecia que los niños presentaron una adherencia inadecuada a la suplementación, en su mayoría con un $67,1 \%$.

Tabla 1. Adherencia a la suplementación con micronutrientes en niños de 6 a 35 meses

$\begin{array}{lll}\text { Adherencia } & \text { Frecuencia } & \text { Porcentaje } \\ \text { Inadecuada } & 53 & 67,10 \% \\ \text { Adecuada } & 26 & 32,90 \% \\ \text { Total } & 79 & 100,00 \%\end{array}$

Fuente: Medición de Adherencia según Directiva Sanitaria $\mathrm{N}^{\mathrm{o}}$ 068-2016 del Ministerio de Salud.

Aplicado a niños de 6 a 35 meses de edad del Puesto de Salud Cono Norte del distrito de Ciudad Nueva, Tacna-2017.

En la Tabla 2, se observa los factores de la madre/cuidador del niño. El grado de instrucción de la madre/cuidador que prevaleció fue el nivel secundario con un $72,2 \%$; respecto a la ocupación el $67,1 \%$ de las participantes eran amas de casa. Concerniente al olvido, el 78,5\% manifestaron olvidarse de dar los micronutrientes algunas veces.

Tabla 2. Factores de la madre/cuidador

\begin{tabular}{lccc} 
& Grado de instrucción & Frecuencia & Porcentaje \\
\hline Primaria & & 11 & $13,9 \%$ \\
Secundaria & 57 & $72,2 \%$ \\
Superior Técnico & & 3 & $3,8 \%$ \\
Superior Universitario & 8 & $10,1 \%$ \\
& Total & 79 & $100,0 \%$ \\
& Ocupación & Frecuencia & Porcentaje \\
Ama de casa & & 53 & $67,1 \%$ \\
Comerciante & 15 & $19,0 \%$ \\
Estudiante & 4 & $5,1 \%$ \\
Otro (policía, enfermera, cosmetóloga, etc.) & 7 & $8,8 \%$ \\
\multicolumn{2}{c}{ Total } & 79 & $100,0 \%$
\end{tabular}


Olvido de administrar el micronutriente

Muchas veces ( 7 a más sobres al mes)

Algunas veces (4 a 6 sobres al mes)

Casi nunca (1 a 3 sobres al mes)

Total

$\begin{array}{cc}\text { Frecuencia } & \text { Porcentaje } \\ 6 & 7,6 \% \\ 62 & 78,5 \% \\ 11 & 13,9 \% \\ 79 & 100,0 \%\end{array}$

Fuente: Cuestionario de Factores Asociados elaborado por Chambilla L. Aplicado a niños de 6 a 35 meses de edad del P.S. Cono Norte del distrito de Ciudad Nueva, Tacna-2017.

La Tabla 3 muestra los factores del micronutriente en el niño. Más de la mitad de las madres/cuidadoras, el $57 \%$, manifestaron que su niño presentó efectos colaterales del micronutriente, siendo el estreñimiento el efecto que más prevaleció en los niños con un 35,4\%. Además, el $43 \%$ de las participantes manifestaron que a su niño no le agradaba consumir el micronutriente.

Tabla 3. Factores del micronutriente

\begin{tabular}{|c|c|c|}
\hline Efectos colaterales & Frecuencia & Porcentaje \\
\hline Si presentó & 45 & $57,0 \%$ \\
\hline No presentó & 34 & $43,0 \%$ \\
\hline $\begin{array}{c}\text { Total } \\
\text { Efectos del micronutriente }\end{array}$ & $\begin{array}{l}79 \\
\text { Frecuencia }\end{array}$ & $\begin{array}{c}100,0 \% \\
\text { Porcentaje }\end{array}$ \\
\hline $\begin{array}{l}\text { Estreñimiento } \\
\text { Diarrea } \\
\text { Nauseas } \\
\text { Ninguno }\end{array}$ & $\begin{array}{c}28 \\
13 \\
4 \\
34\end{array}$ & $\begin{array}{c}35,4 \% \\
16,5 \% \\
5,1 \% \\
43,0 \%\end{array}$ \\
\hline $\begin{array}{c}\text { Total } \\
\text { Aceptación del micronutriente }\end{array}$ & $\begin{array}{l}79 \\
\text { Frecuencia }\end{array}$ & $\begin{array}{l}100,0 \% \\
\text { Porcentaje }\end{array}$ \\
\hline $\begin{array}{l}\text { Le agrada } \\
\text { No le agrada }\end{array}$ & $\begin{array}{l}45 \\
34 \\
79\end{array}$ & $\begin{array}{c}57,0 \% \\
43,0 \% \\
100,0 \%\end{array}$ \\
\hline
\end{tabular}

La tabla 4 muestra los factores de la prestación de servicios de salud presentes en los niños. Donde el $97,5 \%$ de madres manifestaron que el personal de salud sí explicó sobre el consumo de micronutrientes. El 81 \% refirieron haber recibido buen trato por parte del personal de salud. Respecto al tiempo de espera, la mayoría (62\%) manifestó haber sido atendida de inmediato. La disponibilidad del producto fue buena, ya que el $91,1 \%$ de madres/cuidadores manifestaron que en el establecimiento de salud "siempre" contó con el micronutriente.

Tabla 4. Factores de la prestación de servicios de salud

\begin{tabular}{|c|c|c|}
\hline El personal de salud le explica & Frecuencia & Porcentaje \\
\hline Sí & 77 & $97,5 \%$ \\
\hline No & 2 & $2,5 \%$ \\
\hline Total & 79 & $100,0 \%$ \\
\hline Trato del personal de salud & Frecuencia & Porcentaje \\
\hline Bueno & 64 & $81,0 \%$ \\
\hline Regular & 14 & $17,7 \%$ \\
\hline Malo & 1 & $1,3 \%$ \\
\hline $\begin{array}{c}\text { Total } \\
\text { Tiempo de espera }\end{array}$ & $\begin{array}{c}79 \\
\text { Frecuencia }\end{array}$ & $\begin{array}{c}100,0 \% \\
\text { Porcentaje }\end{array}$ \\
\hline Demasiado tiempo (más de 30 min.) & 13 & $16,5 \%$ \\
\hline Poco tiempo (16 - $30 \mathrm{~min})$. & 17 & $21,5 \%$ \\
\hline Me atendieron de inmediato $(0-15 \mathrm{~min})$ & 49 & $62,0 \%$ \\
\hline Total & 79 & $100,0 \%$ \\
\hline
\end{tabular}




$\begin{array}{lccc} & \text { Disponibilidad del micronutriente } & \text { Frecuencia } & \text { Porcentaje } \\ \text { Nunca } & & 0 & 0,0 \% \\ \text { A veces } & & 7 & 8,9 \% \\ \text { Siempre } & \text { Total } & 72 & 91,1 \% \\ & & 79 & 100,0 \%\end{array}$

Fuente: Cuestionario de Factores Asociados elaborado por Chambilla L. Aplicado a niños de 6 a 35 meses de edad del P.S. Cono Norte del distrito de Ciudad Nueva, Tacna-2017

La Tabla 5 muestra la asociación entre la adherencia a la suplementación y los factores de la madre. El análisis estadístico determinó que no existe asociación significativa entre adherencia y grado de instrucción de la madre, de igual forma sucede con la ocupación de la madre y la adherencia donde no se evidenció asociación significativa. Sin embargo, el olvido de dar los micronutrientes fue el único factor que mostró asociación significativa con la adherencia con un p-valor $=0,000$ y un OR $=2,978$ (IC $95 \% 0,988$ $8,980)$.

Tabla 5. Asociación entre adherencia a la suplementación con micronutrientes y factores de la madre/cuidador

\begin{tabular}{|c|c|c|c|c|c|c|c|c|}
\hline \multirow{3}{*}{$\begin{array}{l}\begin{array}{l}\text { Factores de la madre/ } \\
\text { cuidador }\end{array} \\
\text { Grado de instrucción }\end{array}$} & \multicolumn{2}{|c|}{$\begin{array}{l}\text { Adherencia } \\
\text { inadecuada }\end{array}$} & \multicolumn{2}{|c|}{$\begin{array}{l}\text { Adherencia } \\
\text { adecuada }\end{array}$} & \multirow{2}{*}{$\begin{array}{l}\mathrm{X} 2 \\
\mathrm{P}^{*}\end{array}$} & \multirow[t]{2}{*}{$\mathrm{OR}^{* *}$} & \multicolumn{2}{|c|}{ IC $95 \%$} \\
\hline & $\mathrm{n}$ & $\%$ & $\mathrm{n}$ & $\%$ & & & $\mathrm{Li}$ & Ls \\
\hline & & & & & & & & \\
\hline Primaria & 7 & 13,2 & 4 & 15,4 & \multirow{4}{*}{$\begin{array}{c}X^{2}=3,112 \\
P=0,373\end{array}$} & 0,837 & 0,221 & 3,163 \\
\hline Secundaria & 38 & 71,7 & 19 & 73,1 & & 0,933 & 0,326 & 2,674 \\
\hline Superior técnico & 1 & 1,9 & 2 & 7,7 & & 0,481 & 0,029 & 8,006 \\
\hline Superior universitario & 7 & 13,2 & 1 & 3,8 & & 3,804 & 0,443 & 32,689 \\
\hline Total & 53 & 100,0 & 26 & 100,0 & - & - & - & - \\
\hline \multicolumn{9}{|l|}{ Ocupación } \\
\hline Ama de casa & 34 & 64,2 & 19 & 73,1 & & 0,659 & 0,235 & 1,851 \\
\hline Comerciante & 12 & 22,6 & 3 & 11,5 & $X^{2}=1,767$ & 2,244 & 0,573 & 8,781 \\
\hline Estudiante & 3 & 5,7 & 1 & 3,8 & $\mathrm{P}=0,622$ & 1,500 & 0,148 & 15,166 \\
\hline Otro & 4 & 7,5 & 3 & 11,5 & & 0,626 & 0,129 & 3,029 \\
\hline Total & 53 & 100,0 & 26 & 100,0 & - & - & - & - \\
\hline \multicolumn{9}{|l|}{ Olvido } \\
\hline $\begin{array}{c}\text { Casi nunca (1 a } 3 \\
\text { sobres al mes) }\end{array}$ & 2 & 3,8 & 9 & 34,6 & & 0,074 & 0,015 & 0,377 \\
\hline $\begin{array}{l}\text { Algunas veces (4 a } 6 \\
\text { sobres al mes) }\end{array}$ & 45 & 84,9 & 17 & 65,4 & $\begin{array}{c}X^{2}=15,70 \\
P=0,000\end{array}$ & 2,978 & 0,988 & 8,980 \\
\hline $\begin{array}{c}\text { Muchas veces ( } 7 \text { a más } \\
\text { sobres al mes) }\end{array}$ & 6 & 11,3 & 0 & 0,0 & & - & - & - \\
\hline Total & 53 & 100,0 & 26 & 100,0 & - & - & - & - \\
\hline
\end{tabular}

Fuente: Cuestionario de Factores Asociados elaborado por Chambilla L. Aplicado a niños de 6 a 35 meses de edad del P.S.

Cono Norte del distrito de Ciudad Nueva, Tacna-2017.

${ }^{*} \mathrm{p}<0,05$ indica que sí existe asociación entre variables.

**OR, IC $>1$ asociación significativa (factor de riesgo) // OR, IC $<1$ asociación significativa (factor de protección).

La Tabla 6 muestra el análisis estadístico entre las variables, donde se observa que no existe una asociación significativa entre la adherencia y los factores del micronutriente. 
Tabla 6. Asociación entre adherencia a la suplementación con micronutrientes y factores del micronutriente

\begin{tabular}{|c|c|c|c|c|c|c|c|c|}
\hline \multirow{2}{*}{$\begin{array}{c}\text { Factores del } \\
\text { micronutriente }\end{array}$} & \multicolumn{2}{|c|}{$\begin{array}{l}\text { Adherencia } \\
\text { inadecuada }\end{array}$} & \multicolumn{2}{|c|}{$\begin{array}{l}\text { Adherencia } \\
\text { adecuada }\end{array}$} & \multirow{2}{*}{$\begin{array}{l}\mathrm{X} 2 \\
\mathrm{P} *\end{array}$} & \multirow[t]{2}{*}{$\mathrm{OR} * *$} & \multicolumn{2}{|c|}{ IC $95 \%$} \\
\hline & $\mathrm{n}$ & $\%$ & $\mathrm{n}$ & $\%$ & & & $\mathrm{Li}$ & Ls \\
\hline \multicolumn{9}{|c|}{ Presentó efectos colaterales } \\
\hline Sí & 29 & 54,7 & 16 & 61,5 & $X^{2}=0,331$ & 0,756 & 0,290 & 1,967 \\
\hline No & 24 & 45,3 & 10 & 38,5 & $\mathrm{P}=0,565$ & & & \\
\hline Total & 53 & 100,0 & 26 & 100,0 & - & - & - & - \\
\hline \multicolumn{9}{|c|}{ Aceptación del micronutrientes } \\
\hline No le agrada & 22 & 41,5 & 12 & 46,2 & $X^{2}=0,153$ & 0,825 & 0,322 & 2,130 \\
\hline Le agrada & 31 & 58,5 & 14 & 53,8 & $P=0,695$ & & & \\
\hline Total & 53 & 100,0 & 26 & 100,0 & - & - & - & - \\
\hline
\end{tabular}

Fuente:Cuestionario de Factores Asociados elaborado por Chambilla L. Aplicado a niños de 6 a 35 meses de edad del P.S. Cono Norte del distrito de Ciudad Nueva, Tacna-2017

${ }^{*} \mathrm{p}<0,05$ indica que si existe asociación entre variables.

**OR, IC $>1$ asociación significativa (factor de riesgo) // OR, IC $<1$ asociación significativa (factor de protección)

La Tabla 7 muestra la asociación entre la adherencia a la suplementación y los factores de prestación de servicios de salud. Tras el análisis estadístico, se determinó que no existe asociación significativa entre las variables mencionadas.

Tabla 7. Asociación entre adherencia a la suplementación con micronutrientes y factores de la prestación de servicios de salud

\begin{tabular}{|c|c|c|c|c|c|c|c|c|}
\hline \multirow{2}{*}{$\begin{array}{c}\text { Factores de prestación de } \\
\text { servicios de salud } \\
\text { Le explicaron }\end{array}$} & \multicolumn{2}{|c|}{$\begin{array}{l}\text { Adherencia } \\
\text { inadecuada }\end{array}$} & \multicolumn{2}{|c|}{$\begin{array}{l}\text { Adherencia } \\
\text { adecuada }\end{array}$} & \multirow{2}{*}{$\begin{array}{l}\mathrm{X} 2 \\
\mathrm{P} *\end{array}$} & \multirow[t]{2}{*}{ Or** } & \multicolumn{2}{|c|}{ Ic $95 \%$} \\
\hline & $\mathrm{N}^{\mathrm{o}}$ & $\%$ & $\mathrm{~N}^{\mathrm{o}}$ & $\%$ & & & $\mathrm{Li}$ & Ls \\
\hline No & 2 & 3,8 & 0 & 0,0 & $X^{2}=1,007$ & - & - & - \\
\hline Sí & 51 & 96,2 & 26 & 100,0 & $P=0,316$ & & & \\
\hline Total & 53 & 100,0 & 26 & 100,0 & - & - & - & - \\
\hline Malo & 1 & 1,9 & 0 & 0,0 & & - & - & - \\
\hline Regular & 11 & 20,8 & 3 & 11,5 & $\begin{array}{l}X^{2}=1,592 \\
P=0451\end{array}$ & 2,008 & 0,508 & 7,935 \\
\hline Bueno & 41 & 77,4 & 23 & 88,5 & & 0,446 & 0,114 & 1,744 \\
\hline Total & 53 & 100,0 & 26 & 100,0 & - & - & - & - \\
\hline Tiempo de espera & & & & & & & & \\
\hline $\begin{array}{l}\text { Demasiado tiempo (más de } \\
\qquad 30 \mathrm{~min} \text { ) }\end{array}$ & 6 & 11,3 & 7 & 26,9 & & 0,347 & 0,103 & 1,166 \\
\hline Poco tiempo (15 a $30 \mathrm{~min})$ & 12 & 22,6 & 5 & 19,2 & $\begin{array}{c}X=3,093 \\
\mathrm{P}=0,213\end{array}$ & 1,229 & 0,382 & 3,954 \\
\hline $\begin{array}{l}\text { De inmediato } \\
\text { (0 a } 15 \mathrm{~min})\end{array}$ & 35 & 66,0 & 14 & 53,8 & & 1,667 & 0,639 & 4,344 \\
\hline Total & 53 & 100,0 & 26 & 100,0 & - & - & - & - \\
\hline Disponibilidad del micronutri & nte & & & & & & & \\
\hline Nunca & 0 & 0,0 & 0 & 0,0 & & - & - & - \\
\hline A veces & 5 & 9,4 & 2 & 7,7 & $\begin{array}{c}X^{2}=0,066 \\
P=0798\end{array}$ & 1,250 & 0,226 & 6,922 \\
\hline Siempre & 48 & 90,6 & 24 & 92,3 & & 0,800 & 0,144 & 4,430 \\
\hline Total & 53 & 100,0 & 26 & 100,0 & - & - & - & - \\
\hline
\end{tabular}

Fuente:Cuestionario de Factores Asociados elaborado por Chambilla L. Aplicado a niños de 6 a 35 meses de edad del P.S. Cono Norte del distrito de Ciudad Nueva, Tacna-2017

$*_{p}<0,05$ indica que si existe asociación entre variables.

**OR, IC $>1$ asociación significativa (factor de riesgo)// OR, IC $<1$ asociación significativa (factor de protección) 


\section{Discusión}

En la Tabla 1 se aprecia que la mayoría de los niños presentaron una adherencia inadecuada a la suplementación $(67,1 \%)$. Estos resultados son coherentes con el estudio realizado por Munares G. et al. (4) en el 2014, donde se determinó una baja prevalencia de adherencia a los multimicronutrientes $(24,4 \%)$ en niños de 6 a 35 meses en el Perú. Esta adherencia inadecuada genera graves consecuencias en el niño porque al no cumplir con el esquema de la suplementación corremos el riesgo de no cubrir las necesidades de hierro que necesitan en esta etapa crítica de desarrollo cerebral en su primera infancia, lo cual puede generar un deficiente desarrollo cognitivo y motor.

En la Tabla 2 se muestra los factores de la madre/cuidador del niño. Allí, la mayoría de las madres indicó contar con el nivel secundario (72,2\%). Estos resultados son concordantes con el estudio realizado por Christensen et al. (5) en la Red de Salud Pública del Rosario-Argentina en el 2012. Los autores obtuvieron como resultado que el 44,3\% de las madres refirieron no haber terminado la secundaria, siendo este nivel el de mayor prevalencia. Este es un factor que facilita la comprensión al momento de recibir consejería sobre los micronutrientes por el personal de salud. En consecuencia, se favorece la adecuada suplementación $\mathrm{y}$, por ende, se cubren las necesidades de hierro que el niño necesita para su desarrollo cerebral.

Respecto a la ocupación, la mayoría de madres eran amas de casa $(67,1 \%)$. Estos resultados coinciden con el estudio realizado por Munayco et al. (6), en tres regiones andinas del Perú en el 2013. En referido estudio, se determinó que el 84,4 \% de las madres eran amas de casa. El hecho de que las madres del Puesto de Salud Cono Norte se encuentren en el hogar la mayor parte del día facilita la administración del micronutriente al niño, ya que pueden administrarlos en una de sus comidas principales.

Respecto al olvido en la administración de micronutrientes, la mayoría de madres/cuidadores $(78,5$ \%) manifestaron olvidarse de dar los micronutrientes algunas veces. Estos resultados coinciden con el estudio realizado por la Fundación de Acción Contra el Hambre y el Fondo de las Naciones Unidas (7), en los departamentos de Huancavelica, Apurímac y Ayacucho en el 2013. El estudio tuvo como resultado que las madres se olvidaban de preparar las chispitas a los niños y no se las daban. Podemos deducir que las madres en estudio se olvidaban de dar los micronutrientes porque no tenían muy claro la finalidad de los micronutrientes; además, es muy probable que la importancia que le atribuían a los micronutrientes no era significativa, llegando a existir la posibilidad de que las madres/cuidadores hayan considerado abandonar la suplementación. Asimismo, las madres/cuidadores al saber que sus niños no tenían anemia no consideraban necesario la administración del micronutriente porque no existía una razón grave para que su niño lo consuma. Estos factores fueron desfavorables para que los niños hayan sido suplementados.

En la Tabla 3 se muestran los factores del micronutriente en el niño. Se observa que más de la $\operatorname{mitad}(57 \%)$ de madres/cuidadores manifestaron que su niño ha presentado efectos colaterales del micronutriente. Siendo el estreñimiento el efecto que más prevaleció. Tales resultados fueron similares con el estudio realizado por Castro-Flores M. (8) en Huachi Grande-Ecuador en el 2011. En el estudio se determinó que la mayoría de niños presentó efectos adversos, siendo principalmente la diarrea con un 38,33\% y el estreñimiento con un $30 \%$. Aunque los micronutrientes contienen el hierro en forma de fumarato ferroso encapsulado para ocultar los malestares gastrointestinales (9), estos malestares se evidenciaron en los niños, lo cual generó cierta desconfianza en las madres/cuidadores, pudiendo llegar hasta la suspensión del micronutriente. Es por ello necesario dar a conocer los posibles efectos colaterales que puede producir el micronutriente a las madres; también, es importante indicar que estos efectos son leves y pasajeros, así como los cuidados frente a los efectos colaterales para poder así disminuir la preocupación y temor de los padres y a la vez reducir el riesgo de que suspendan la suplementación.

Existe un porcentaje significativo de niños con desagrado al consumo de los micronutrientes (43\%). Los resultados coincidieron con el estudio de Sammartino G. (10) en Buenos Aires en el 2010. En referido estudio, los autores llegaron a la conclusión de que algunos niños rechazan la ingesta de hierro por las desagradables sensaciones (escupen, lloran) que produce. Es probable que los alimentos preparados por las madres/cuidadores no se den inmediatamente al niño o la temperatura de los alimentos no sea la adecuada, produciendo alteraciones en el sabor y color de los alimentos, conllevando así a que el niño escupa y ya no desee comer los alimentos que contienen el micronutriente. Por ello, es necesario recalcar la importancia de una preparación adecuada del micronutriente para facilitar su consumo y brindar alternativas de administración del micronutriente en caso de rechazo.

En la Tabla 4 se muestran los factores de la prestación de servicios de salud a las madres/cuidadores participantes. En donde apreciamos que el personal de salud sí explica sobre el consumo de micronutrientes según refirieron las madres/cuidadores $(97,5 \%)$. Estos 
resultados son similares al estudio realizado por JuncoGuillermo J. (11) en Ayacucho en el 2015. En referido estudio se obtuvo como resultado que el $90 \%$ de las madres refirieron haber recibido orientación para la preparación del multimicronutriente. Es importante que la madre/cuidador conozca la importancia y el consumo adecuado del micronutriente, para despertar el interés y su participación activa en la suplementación del niño. Por ello, el personal de salud debe estandarizar la información a brindar para que esta sea de calidad y las madres sepan con precisión la utilidad del micronutriente.

Respecto al trato del personal de salud, las madres/cuidadores refirieron haber recibido un buen trato por parte del personal de salud (81 \%). Los resultados son coherentes con el estudio realizado por Hinostroza F. (12) en Lima en el 2015. En tal estudio, los autores concluyeron que todas las madres consideraron que el personal de salud que les atiende en la entrega del multimicronutriente fue amable, paciente, brindó confianza para poder preguntar dudas y respondió a las preguntas realizadas. Este factor permite establecer una estrecha relación con las madres/cuidadores en el momento de la consulta para concientizar e impulsar la participación activa de la madre/cuidador en la suplementación de su niño.

Respecto al tiempo de espera, la mayoría de las participantes $(62 \%)$ manifestó haber sido atendida de inmediato. Los resultados difieren con la investigación de Luna-Guerrero B. (13) en Lima en el 2013. En referido estudio se obtuvo como resultado que el $75 \%$ de madres consideraron que el tiempo de espera para la atención es muy prolongado. El hecho de ser atendido de inmediato, es un factor que favorece la continuidad y cumplimiento de la suplementación del niño y evita molestias en las madres/cuidadores.

La disponibilidad del micronutriente en el Puesto de Salud Cono Norte es buena según manifestaron las madres en estudio (91,1 \%). Los resultados coincidieron con la investigación de JuncoGuillermo J. (11) realizado en Ayacucho en el 2015. El autor concluyó que las madres refirieron que el personal de los establecimientos de salud cumplía con entregarles los 30 sobres mensuales. Una buena organización y abastecimiento de insumos en el establecimiento facilita el cumplimiento de la suplementación.

En la Tabla 5, los análisis estadísticos determinaron que el olvido es el único factor que tiene una asociación significativa con la adherencia. Se puede considerar al olvido como un factor de riesgo para la adherencia, entendiendo que el riesgo de presentar adherencia inadecuada es dos veces mayor en aquellos niños cuyas madres se olvidan de dar los micronutrientes a diferencia de las que no se olvidan. Los resultados son coherentes con el estudio realizado por Espichan-Ávila P. (14), quien realizó un trabajo de investigación en San Martín de Porres en la ciudad de Lima en el 2013. El mencionado estudio determinó que el olvido es uno de los factores más influyentes en la adherencia (90\%). Asimismo, los resultados coinciden también con la investigación de Christensen et al. (5) en Santa Fe en Argentina en el 2012. En el estudio se concluye que el motivo más frecuente para la falta de adherencia fue el olvido de la madre. El olvido viene a constituir un factor de riesgo para la adherencia; por lo tanto, debemos intervenir arduamente en él, sensibilizando a las madres acerca de temas como la importancia de la suplementación en sus niños, las consecuencias de la anemia en el crecimiento y desarrollo, así como el compromiso en la participación activa durante todo el periodo de la suplementación para lograr una adherencia adecuada. El olvido de la madre/cuidador interrumpe la continuidad en el consumo de los micronutrientes y, por ende, disminuye la dosis que el niño necesita ingerir para cubrir sus requerimientos diarios de hierro; ya que en esta primera infancia, el hierro es un elemento esencial para la etapa crítica del desarrollo cerebral del niño tanto en la mielinización como en la sinapsis neuronal.

\section{Conclusiones}

- La adherencia a la suplementación con micronutrientes en los niños fue inadecuada en su mayoría.

- Los factores identificados fueron: las madres cuidadoras de los niños en su mayoría contaban con grado de instrucción secundaria, eran amas de casa y se olvidaban de dar los micronutrientes. Respecto a los micronutrientes, más de la mitad de los niños presentó efectos colaterales, siendo el estreñimiento el de mayor frecuencia; además, a casi la mitad de niños no les agradaba consumir el micronutriente. Concerniente a la prestación de servicios de salud, el personal de salud explicaba sobre el consumo de micronutrientes, su trato fue bueno, la atención fue inmediata y la disponibilidad del micronutriente fue buena.

- La adherencia se asoció significativamente con el olvido de dar los micronutrientes. 


\section{Referencias bibliográficas}

1. World Health Organization. The Global The Prevalence of Anaemia in 2011 [internet]. Geneva: World Health Organization; 2015 [citado el 20 de diciembre del 2016]. Disponible en: http://apps.who.int/iris/ bitstream/ 10665/177094/1/9789241564960_eng.pdf?ua=1

2. Ministerio de Salud del Perú. Plan Nacional de Reducción y Control de la Anemia y la Desnutrición Crónica Infantil en el Perú: 2017-2021[internet]. Lima; 2016 [Citado el 11 de Febrero del 2017] D i s p o n i b 1 e e $n$ : http://www.minsa.gob.pe/portada/Especiales/2016/ anemia/documentos/1\%20Plan\% 20Anemia.pdf

3. Ministerio de Salud. Directiva Sanitaria para la prevención de anemia mediante la suplementación con micronutrientes y hierro en niñas y niños menores de 36 meses [internet]. Lima: Sinco Editores SAC; 2016

4. Munares G, Gomez G. Adherencia a multimicronutrientes y factores asociados en niños de 6-35 meses de sitios centinela, Ministerio de Salud, Perú. Revista Brasileira de Epidemiología [Internet], 2016 [citado el 24 de enero del 2017]; 19 (3).

5. Christensen L, Sguassero Y, Cuesta C. Anemia y adherencia a la suplementación oral con hierro en una muestra de niños usuarios de la Red de Salud Pública de Rosario, Santa Fe. Arch. Argent. Pediatr [Internet], 2013 [citado el 12 de marzo del 2017]; $111(4)$

6. Munayco C, Ulloa M, Medina J, Lozano C, Tejada V, Castro C. Evaluación del impacto de los multimicronutrientes en polvo sobre la Anemia infantil en tres regiones andinas del Perú. Rev Peru Med Exp Salud Pública [Internet]. 2013 [citado el 12 de febrero del 2017]; 30 (2).

7. Fundación de Acción Contra el Hambre, Fondo de las Naciones Unidas. Valores, saberes y experiencias en el consumo de chispitas en los departamentos de Huancavelica, Apurímac y Ayacucho. [Internet]. Lima: Fundación de Acción Contra el Hambre; 2013 [citado el 20 de febrero del 2017].

8. Rodríguez A, Gómez A. Factores influyentes en adherencia al tratamiento en pacientes con riesgo cardiovascular. Avances en Enfermería [Internet].
2010 [citad el 3 de mayo del 2017]; 28(1).

9. Grullon R. Micronutrientes espolvoreados (chispitas nutricionales) una nueva alternativa para combatir anemia y otras deficiencias de micronutrientes en niños [Internet]. 2015 [citado el 26 de abril del 20017 . D i s p o n i b le e n : http://slideplayer.es/slide/3206787/

10. Sammartino G. Representaciones culturales acerca de la anemia y la suplementación de hierro. Archivos Argentinos de Pediatría [Internet], 2010 [citado el 5 de mayo del 2017]; 108 (5).

11. Guillermo J. Identificación de los factores que contribuyen y limitan la efectividad del programa de suplementación con multimicronutrientes en reducción de la anemia de niños menores de 3 años del ámbito rural de Vinchos de Ayacucho [Tesis en internet]. Lima: Pontificia Universidad Católica del Perú; 2015 [citado el 3 de febrero del 2017]. Disponible en: http://tesis.pucp.edu.pe/repositorio/ handle/123456789/6650

12. Hinostroza F. Barreras y motivaciones en el nivel de adherencia a la suplementación con multimicronutrientes en madres de niños menores de 36 meses, cercado de Lima [Tesis en internet]. Lima: Universidad Nacional Mayor de San Marcos; 2015 . D i s p o n i b le e n : http://cybertesis.unmsm.edu.pe/handle/cybertesis/ 4226

13. Luna-Guerrero B. Factores que influyen en la adherencia al tratamiento de anemia ferropénica en niños menores de 3 años del Centro de Salud Ex Fundo Naranjal [Tesis en internet] Lima:Universidad nacional Mayor de San Marcos; 2013 [citado el 27 de marzo del 2017]. Disponible en:http://ateneo.unmsm.edu.pe/ateneo/bitstream/1 $23456789 / 4494 / 1 /$ Luna Guerrero Beatriz Veronica 2014.pdf

14. Espichan-Avila P. Factores de adherencia a la suplementación con sprinkles asociados al incremento de hemoglobina en niños de 6 a 60 meses, de asentamientos humanos del Distrito de San Martín de Porres [Tesis en internet]. Lima: Universidad Nacional Mayor de San Marcos; 2013 [citado el 25 de febrero del 2017]. Disponible en: http://cybertesis.unmsm.edu.pe/bitstream/ cybertesis $/ 3417 / 1 /$ Espich\%C3\%A1n_ap.pdf
Correspondencia

lucesita_mph@hotmail.com
Fecha de recepción: 07 de julio de 2019

Fecha de aceptación: 15 de noviembre de 2019 